\title{
High Titer Anti-Basement Membrane Antibodies in a Subset of Patients with Pediatric Systemic Lupus Erythematosus
}

\author{
Alvaro Orjuela $^{\mathrm{a}, \mathrm{d}}$ Adisak Suwanichkul $^{\mathrm{a}, \mathrm{d}}$ Debra Canter ${ }^{\mathrm{a}, \mathrm{e}}$ Charles G. Minard ${ }^{\mathrm{c}}$ \\ Sridevi Devaraj ${ }^{b, f}$ M. John Hicks ${ }^{b, f}$ Eyal Muscal ${ }^{a, e}$ Scott E. Wenderfera, ${ }^{a}$ \\ ${ }^{a}$ Department of Pediatrics, ${ }^{b}$ Pathology, and ${ }^{c}$ Dan L. Duncan Institute for Clinical and Translational Research, \\ Baylor College of Medicine, and ${ }^{\mathrm{d}}$ Renal, ${ }^{e}$ Rheumatology, and ${ }^{\mathrm{f} P a t h o l o g y}$ Sections, Texas Children's Hospital, \\ Houston, Tex., USA
}

\section{Key Words}

Glomerulonephritis · Pediatrics · Inflammation

\begin{abstract}
Background/Aims: There is a critical need for more noninvasive biomarkers to identify nephritis in patients with systemic lupus erythematosus (SLE). Recent studies in a model mouse and an adult SLE patient cohort suggest that antibasement membrane antibody levels correlate well with lupus activity and kidney injury. The purpose of this study was to assess the anti-basement membrane reactivity in pediatric SLE (pSLE) patients with or without nephritis. Methods: Auto-antibodies to basement membrane antigens were assessed using an anti-matrigel ELISA. Endpoint titers were measured in pSLE patients and healthy children, as well as in autoimmune and non-immune mice, with good reproducing capabilities. Findings were also analyzed with respect to the presence or absence of nephritis, dsDNA antibodies, and other manifestations of pSLE. Results: MRL/lpr mice developed high-titer anti-matrigel antibodies, whereas C57BL/6 mice did not. In a cohort of $21 \mathrm{pSLE}$ patients and 22 pediatric controls, high-titer anti-matrigel $\lg G, \lg M$ and $\lg A$ antibody levels were specific for pSLE. High-titer anti-matrigel lgG3 levels could distinguish with good sensitivity the 13 pSLE patients with a history of nephritis from the 8 non-renal pSLE
\end{abstract}

patients. High-titer anti-matrigel $\lg G, \lg A, \lg M$ or $\lg G 3$ did not correlate with positive anti-double stranded DNA, but defined an overlapping subset of patients. Conclusion: The addition of anti-basement membrane antibody testing to serologic testing in pSLE may help to monitor disease activity or to define important subsets of patients with risks for specific disease manifestations.

(c) 2015 S. Karger AG, Basel

\section{Introduction}

Intense efforts to develop diagnostic tools to detect the presence of nephritis in systemic lupus erythematosus (SLE) [1-4] have been made. But the need is still greatly felt in pediatric patients with SLE because the prevalence and severity of nephritis are greater in children than in adults [5]. Hypocomplementemia, as measured by $\mathrm{CH} 50$ is $70 \%$ sensitive and 70\% specific for SLE, low C3 levels are 64\% sensitive and $91 \%$ specific, and low C4 levels are $64 \%$ sensitive and 65\% specific for SLE diagnosis [6]. The use of proteinuria and creatinine clearance as markers for renal disease activity is controversial. Persistent proteinuria can be caused by acute or chronic lesions, and does not necessarily reflect ongoing inflammation in the kidneys. Kidney flares can occur before renal functions decline by available

\section{KARGER 125}

(c) 2015 S. Karger AG, Base

0250-8095/15/0413-0241\$39.50/0

E-Mail karger@karger.com

www.karger.com/ajn
Scott E. Wenderfer

Renal Section, Texas Children's Hospital

1102 Bates Avenue, Ste. 245

Houston, TX-77030 (USA)

E-Mail wenderfe@bcm.edu 
laboratory parameters [7]. Several scoring systems based on combinations of clinical parameters, such as SLEDAI and BILAG, have been developed and validated in clinical trials, but have not been widely used to predict either nephritis risk or response to therapy in clinical practice.

Several candidate urinary biomarkers have also been studied for the monitoring of kidney inflammation in pSLE. One study in adults and children reported that a combination of elevated urinary MCP-1, ceruloplasmin, al-acid glycoprotein, and NGAL was predictive of a more active nephritis (area under the curve (AUC) 0.85), whereas elevated MCP-1 and NGAL were together more predictive of chronic renal injury (AUC 0.83) [8]. A prospective pediatric study demonstrated that either urinary MCP-1 or NGAL could discriminate between active renal lupus and non-renal pSLE with an AUC value 0.81 $(\mathrm{p}=0.013)$ and $0.76(\mathrm{p}=0.003)$, respectively [9]. These markers and others are being confirmed in larger cohorts, but additional markers will be needed to be added to these panels in order to achieve sufficient accuracy (AUC >0.9) required to help guide $\mathrm{LN}$ therapy.

Others have focused on subsets of auto-antibodies (Abs) that either cause nephritis or serve as markers for the presence of nephritis in SLE. The subset of auto-Abs that is used by most in the clinic to monitor for renal disease is the one with reactivity against anti-double stranded DNA [10]. Serum titers of anti-dsDNA Abs are routinely used clinically to monitor for renal flares and response to therapy. In a meta-analysis performed by the American College of Rheumatology's ad hoc Committee on Immunologic Testing Guidelines, assays measuring anti-dsDNA Abs predicted a diagnosis of SLE with a weighted mean sensitivity of $57 \%$ and specificity of $97 \%$ [10]. The presence of high-titer anti-dsDNA Abs predicted the presence of active renal disease in SLE patients with a weighted mean sensitivity of $86 \%$ and a specificity of $45 \%$. Titers of anti-dsDNA Abs correlate with the degree of renal injury in SLE, but only to a limited extent [10].

Recently, there has been renewed interest in antibasement membrane (BM) Abs, due to new findings reported in the NZB/W F1 mouse model of lupus [4]. This model displays loss of tolerance, auto- $\mathrm{Ab}$ generation, and inflammatory kidney injury comparable to that seen in patients with SLE. Genetic variation in the F1 mice leads to variable production of auto-Abs of varying specificities that correspond in differing degrees of nephritis [11]. Anti-dsDNA Ab titers are not predictive of subsequent nephritis in the NZB/W F1. However, among 69 monoclonal Abs originating from the mouse strain, there was a perfect correlation between Abs that bound to BM antigens with high affinity in vitro and those that accumulated in glomeruli and caused significant proteinuria after injection into non-immune mice [4]. An ELISA was used with matrigel as a surrogate for detecting mouse Abs that were bound to BM antigens. Although antimatrigel $\mathrm{Ab}$ titers have not been rigorously tested as a diagnostic tool in human SLE, there is some promising data. Multiplex analysis of circulating auto-Abs in a single-center cohort of 37 adults with SLE showed a correlation between the presence of high IgG titer anti-matrigel Abs, anti-DNA Abs (ssDNA, dsDNA, chromatin), and higher total and renal SLE disease activity scores (SLEDAI scores) [12].

In order to determine whether heightened reactivity to $\mathrm{BM}$ antigens occurs in pediatric SLE patients, reactivity to matrigel in human plasma and serum was developed. Children with and without lupus were tested to assess whether a correlation exists between anti-BM Ab titer and a clinical diagnosis of pSLE, a history of nephritis, or reactivity to anti-dsDNA. Titers of IgG, IgM, IgA and Ig3 anti-BM Abs were measured in all samples. Relationships between ACR criteria and clinical findings were also explored.

\section{Methods}

Matrigel (BD Biosciences) was used as a surrogate BM preparation. Matrigel is derived from an EHS sarcoma cell line and is composed primarily of laminin (55\%), collagen type IV (30\%), entactin (8\%), and heparin sulfate proteoglycans (5\%). An anti-matrigel ELISA for detection of mouse Abs was performed as previously described [4]. Briefly, high protein-binding 96-well microtiter plates (Nunc Apogent) were coated with Matrigel at $500 \mu \mathrm{g} /$ well in cold PBS $\left(4^{\circ} \mathrm{C}\right)$. After gelling at $4^{\circ} \mathrm{C}$ overnight, serum or plasma samples were added at 2 -fold serial dilutions. Bound immunoglobulin from samples was detected using a biotin-conjugated goat anti-mouse IgG polyclonal Ab (Invitrogen) diluted 1:20,000, followed by streptavidin-HRP (R\&D Systems) diluted 1:500, and TMB substrate (Pierce). Optical density was measured at $450 \mathrm{~nm}$ (OD 450) using a SpectraMax i3 plate reader (Molecular Devices). The protocol was changed for detection of anti-matrigel Abs in humans by substituting anti-human IgG polyclonal Ab (Invitrogen), also diluted at 1:20,000. For anti-matrigel IgA, IgM, and IgG3 ELISAs, the same protocol was followed, except for the use of a biotin-conjugated goat anti-human IgA diluted at 1:5,000, antihuman IgM diluted at 1:2,000, or anti-human Ig-G3 diluted at 1: 1,000 (Invitrogen). Testing of individual patient samples on separate occasions by two different experimenters (A.O. and A.S.) produced results that were highly reproducible with low inter-assay variation (CV 10-15\%). Endpoint titers were determined as the dilution at which binding was no greater than the non-specific binding measured from normal human serum.

For anti-dsDNA Ab ELISA, plates were coated in poly-L-lysine $5 \mu \mathrm{g} / \mathrm{well}$, and then with $10 \mu \mathrm{g} / \mathrm{ml}$ calf thymus DNA (Sigma) pretreated with $\mathrm{S} 1$ nuclease for $30 \mathrm{~min}$ at $37^{\circ} \mathrm{C}$ to remove any single 
stranded DNA. Wells were then blocked with 5\% BSA/PBS. After washing, serial plasma dilutions were added. After a 2-hour incubation at room temperature, plates were washed and horseradish peroxidase-conjugated detection Ab was added (anti-human IgG; Jackson Immunoresearch), diluted in the ratio 1:20,000. TMB substrate was added, OD 450 was measured, and endpoint titers were calculated in comparison with pooled negative control plasma. A positive test was defined as an endpoint titer $>1: 40$.

\section{Mouse Studies}

Serum was obtained from 24-week-old female MRL/lpr (age where all mice develop immune complex nephritis and $>50 \%$ die from kidney failure) and age-matched female C57BL/6 mice. C57BL/6 mice are maintained in the Baylor College of Medicine (BCM) animal colony. MRL/lpr sera was a kind gift from Dr. Michael C. Braun (at BCM). All animal studies were performed after approval from BCM's Institutional Animal Care and Use Committee.

\section{pSLE Patients}

Serum samples were obtained from 21 unique pSLE patients enrolled in a longitudinal study of cognitive impairment in pSLE (June 2008-September 2011) [13]. Eligibility criteria included: age $\leq 18$-years at the time of enrollment and $\leq 16$-years at the time of diagnosis, and diagnosis of SLE according to the revised 1997 American College of Rheumatology criteria [14]. Samples from subjects with acute kidney injury due to etiologies other than autoimmune nephritis (pyelonephritis, acute tubular necrosis, or acute interstitial nephritis) were excluded.

Consent was obtained for reviewing the medical records of 10 of the 21 study subjects. Demographics and lab results were obtained from clinical encounters within 2 months of sample collection. Renal function was assessed by serum creatinine or 24-hour creatinine clearance. Estimated GFR was calculated for each patient using the Schwartz formula [15]. Lupus activity was assessed by the levels of complement components C3 and C4 and anti-dsDNA and anti-RNP Abs titers. Renal involvement was assessed in terms of proteinuria, as measured by 24 -hour urine collection and/ or urine protein creatinine ratio on random urine samples, and hematuria, as recorded by dipstick, urinalysis, and urinary sediment analysis. All human studies were performed in accordance with the Declaration of Helsinki after approval by BCM's Institutional Review Board.

\section{Healthy Controls}

Control sera from 22 healthy children without renal failure were obtained from a bio-repository maintained at TCH. Eligibility criteria included age $\leq 18$, and no known diagnosis of any form of autoimmune disease.

\section{Statistical Analysis}

Statistical analyses were performed using Sigma Plot software version 11 (www.systat.com) and SAS software version 9.3 (SAS Institute Inc., Cary, N.C., USA). Continuous data were expressed as means with standard deviations or standard errors, medians with ranges, or frequencies with percentages as appropriate. Fisher's exact was used to compare proportions between groups and the Mann-Whitney test was used to evaluate differences in continuous data between groups. General linear mixed models were used to examine the relationship between OD 450 levels at varying dilutions for each patient group (normal controls, pSLE patients with nephritis, and pSLE patients without nephritis) taking into account the nature of repeated sampling for some pSLE patients. The AUC of the receiver operating characteristic (ROC) curve was calculated to examine the ability of the endpoint titer to distinguish between specific groups of patients, and Youden's index was calculated to determine the cut point for defining high-titer Abs. In addition, the sensitivity and specificity of cutoff values for defining high and low endpoint titers were calculated. Summary statistics are presented as medians with ranges due to the small sample size. Finally, a general linear mixed model was used to compare interval clinical characteristics between pSLE patients with either low or high endpoint titers. A p value $<0.05$ was considered statistically significant for all analyses.

\section{Results}

\section{Sera from Autoimmune MRL/lpr Mice Bind to} Matrigel at Higher Titers Than Non-Autoimmune B6 Mice Sera

In order to confirm the previous findings of anti-BM reactivity in murine lupus models, anti-matrigel ELISA [4] was used to test sera from 6-month-old female MRL/ lpr and non-autoimmune C57BL/6 mice. By 6-months of age, female MRL/lpr mice all have high-titer anti-dsDNA antibodies, lymphadenopathy, glomerulonephritis, and some degree of kidney failure. An increase in endpoint titer in MRL/lpr mice corresponded with comparable increases in OD 450 at 1:20 and 1:40 dilution of the serum samples. Results demonstrated that MRL/lpr mice had developed anti-matrigel $\mathrm{Ab}$ titers that were 10 to 20 -fold higher than non-autoimmune mice (fig. 1a).

\section{Anti-Matrigel Antibody Titers as Predictor for $p S L E$}

Next, the ELISA protocol was modified to detect human IgG and used to measure anti-BM reactivity in a cohort of 21 unique pediatric SLE patients and 22 agematched, non-SLE controls. A majority of non-SLE controls had endpoint titers of 1:40 or lower and this was designated as the cut-off for high-titer anti-matrigel $\mathrm{Ab}$ levels (fig. 1b). Of the 21 pSLE patients tested, 11 had endpoint titers $>1: 40$ and were considered high-titer. Analysis of the ROC curve for prediction of pSLE by presence of high-titer anti-matrigel IgG revealed an AUC of 0.66 (95\% CI: 0.48, 0.84; fig. 1c), which is comparable to that of anti-dsDNA IgG and other subsets of anti-nuclear antibodies in the literature. The specificity of high-titer antimatrigel IgG Abs for detecting pSLE was measured to be $91 \%$ with a sensitivity of $52 \%$, using the definition of endpoint titer $>1: 40$ (table 1 ). 


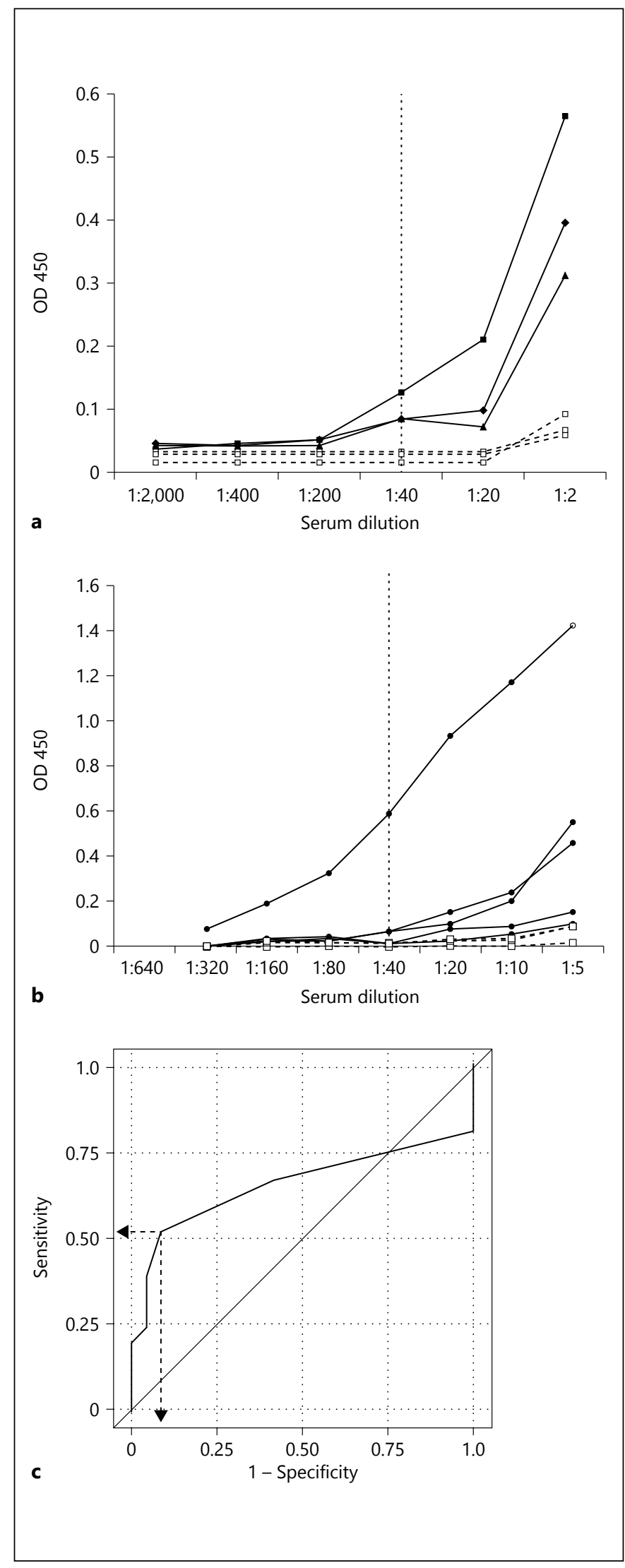

A general linear mixed model was constructed to compare OD 450 measures at each dilution between pSLE patient samples and non-SLE samples. There were no statistically significant differences in OD 450 measures between PSLE and non-SLE samples for any dilution (data not shown). For this reason, endpoint titers were selected as the best strategy to characterize individuals with hightiter anti-matrigel Abs.

To determine if anti-matrigel IgA or IgM antibodies were better at predicting pSLE than anti-matrigel IgG, the ELISA protocol was modified and the same pSLE and non-SLE samples were tested. There are 4 different IgG isotypes, and the anti-matrigel Ab ELISA was successfully adapted for the detection of one of these subsets, IgG3. Results were normalized such that the cut-offs for hightiter Abs were also >1:40. By Fisher's exact test, all 3 isotypes of anti-matrigel Abs were present at high titer in a significantly greater proportion of pSLE patients than in non-SLE control children (table 1). Anti-matrigel IgA an-

Table 1. High-titer anti-matrigel antibodies ${ }^{1}$ occur more commonly in SLE than non-SLE cohorts, and pSLE patients with a history of nephritis tended to have high-titer IgG3 antibodies

\begin{tabular}{|c|c|c|c|c|c|c|}
\hline \multirow[t]{2}{*}{$\begin{array}{l}\text { Ig } \\
\text { isotype }\end{array}$} & \multicolumn{3}{|c|}{$\begin{array}{l}\text { pSLE }(\mathrm{n}=21) \text { vs. } \\
\text { non-SLE }(\mathrm{n}=22)\end{array}$} & \multicolumn{3}{|c|}{$\begin{array}{l}\text { History of nephritis }(\mathrm{n}=13) \text { vs. } \\
\text { non-renal SLE }(\mathrm{n}=8)\end{array}$} \\
\hline & $\begin{array}{l}\text { sensitivity } \\
(\%)\end{array}$ & $\begin{array}{l}\text { specificity } \\
(\%)\end{array}$ & $\begin{array}{l}\mathrm{p} \\
\text { value }^{2}\end{array}$ & $\begin{array}{l}\text { sensitivity } \\
(\%)\end{array}$ & $\begin{array}{l}\text { specificity } \\
(\%)\end{array}$ & $\begin{array}{l}\mathrm{p} \\
\text { value }^{2}\end{array}$ \\
\hline $\operatorname{IgG}$ & 52 & 91 & 0.003 & 42 & 33 & 0.371 \\
\hline IgA & 29 & 95 & 0.046 & 38 & 88 & 0.342 \\
\hline IgM & 66 & 78 & 0.005 & 62 & 25 & 0.656 \\
\hline IgG3 & 42 & 81 & 0.168 & 88 & 64 & 0.059 \\
\hline
\end{tabular}

Fig. 1. Autoimmune MRL/lpr mice and a subset of children with SLE develop high-titer anti-matrigel antibodies. Representative ELISA results from serial dilutions of (a) sera from 3 separate 6-month-old female MRL/lpr mice with nephritis (black circles) and 3 separate age-matched female non-autoimmune C57BL/6 mice (white squares), or (b) plasma from separate children with SLE (black circles) and age matched non-SLE controls (white squares). Samples were tested in triplicate and results are shown as mean OD. Samples requiring dilution greater than 1:40 to reach endpoint were considered to have a high-titer anti-matrigel Abs. c ROC curve using IgG anti-matrigel endpoint titers to predict the presence of SLE in children (SLE, $\mathrm{n}=21$; non-SLE, $\mathrm{n}=22$ ). $\mathrm{AUC}=$ 0.656 . The dotted line represents the cut point indicated by Youden's index, with $91 \%$ specificity and $52 \%$ sensitivity, used for table 1 .
Orjuela/Suwanichkul/Canter/Minard/ Devaraj/Hicks/Muscal/Wenderfer 
tibodies were the most specific for discriminating between pSLE and non-SLE samples, but were the least likely isotype to be present in high titer.

\section{Anti-Matrigel Antibody Titers Can Subdivide Groups of pSLE Patients}

Thirteen of the 21 pSLE patients tested had a history of lupus nephritis. In order to see if a high titer of antimatrigel Abs could discriminate between pSLE patients with a history of nephritis and non-renal pSLE patients, a subset analysis was performed. General linear mixed modeling was used to compare OD measures for the IgG ELISA at each serum dilution, and we found no significant differences. There was also no statistically significant difference in endpoint titers using the $\operatorname{IgA}$, IgG, or IgM ELISAs (table 1); however, the specificity of high-titer IgA anti-matrigel Abs was $88 \%$. Patients with a history of nephritis tended to have higher IgG3 titers than non-renal pSLE patients.

In order to see if reactivity to matrigel was simply a surrogate measure of anti-dsDNA reactivity in our pSLE cohort, all samples were tested by an anti-dsDNA $\mathrm{Ab}$ ELISA [16]. In this cohort, high-titer dsDNA IgG Abs did not distinguish between pSLE with a history of nephritis and non-renal pSLE (specificity 50\%, sensitivity $62 \%$, $\mathrm{p}=0.67)$. Results indicated that the presence of high-titer anti-matrigel IgG, IgA, IgM, or IgG3 did not correlate with the occurrence of positive anti-dsDNA IgG Abs (table 2; $\mathrm{p}=1.0$ for $\operatorname{IgG}$, IgM and IgG3, and $\mathrm{p}=0.37$ for $\operatorname{IgA}$ ).

Table 2. Presence of high-titer anti-matrigel antibodies does not associate with the presence of anti-double stranded DNA antibodies in children with SLE $^{1}$

\begin{tabular}{|c|c|c|}
\hline & \multicolumn{2}{|c|}{ IgG anti-dsDNA Abs } \\
\hline & positive & negative \\
\hline \multicolumn{3}{|c|}{ IgG anti-matrigel $\mathrm{Ab}$ titer (sensitivity $67 \%$, specificity $56 \%$ ) } \\
\hline High & 8 & 4 \\
\hline Low & 4 & 5 \\
\hline \multicolumn{3}{|c|}{ IgM anti-matrigel $\mathrm{Ab}$ titer (sensitivity $62 \%$, specificity $50 \%$ ) } \\
\hline High & 8 & 5 \\
\hline Low & 4 & 4 \\
\hline \multicolumn{3}{|c|}{ IgA anti-matrigel $\mathrm{Ab}$ titer (sensitivity $75 \%$, specificity $54 \%$ ) } \\
\hline High & 6 & 2 \\
\hline Low & 6 & 7 \\
\hline \multicolumn{3}{|c|}{ IgG3 anti-matrigel $\mathrm{Ab}$ titer (sensitivity $50 \%$, specificity $45 \%$ ) } \\
\hline High & 4 & 4 \\
\hline Low & 6 & 5 \\
\hline
\end{tabular}

${ }^{1}$ High reactivity defined as endpoint titers $>1: 40$.
There were 8 pSLE patients who were anti-dsDNA $\mathrm{Ab}$ positive and high-titer anti-matrigel IgG, 4 double-positive patients with high IgG3, 6 double-positive patients with high IgA, and 8 double-positive patients with hightiter IgM anti-matrigel Abs. Only testing positive for both anti-dsDNA Abs and anti-matrigel IgA was significantly associated with a history of nephritis $(\mathrm{p}=0.046)$.

Clinical data were available for 10 of the pSLE patients. All 10 patients were female and predominately Hispanic $(80 \%)$. Six of the patients had a history of lupus nephritis diagnosed by renal biopsy, and these patients tended to be younger (median 14.5 vs. 17-year-old). The relationship between high-titer anti-matrigel Abs and each ACR (American College of Rheumatology) criterion for SLE was investigated (online suppl. table 1; for all online suppl. material, see www.karger.com/doi/10.1159/ 000381965), but the presence of high-titers did not distinguish between the presence or absence of any criterion. High-titer IgG and IgM anti-matrigel Abs tended to be less common in patients who at one point developed discoid rash. High-titer anti-matrigel IgA Abs were also more common in pSLE patients with a history of photosensitivity or mouth ulcers, and less common in those with a history of hematologic or neurologic disorder. In contrast, high-titer anti-matrigel IgG3 Abs were more commonly detected in patients who at one point developed kidney disorder, and less common in those with history of photosensitivity or mouth ulcers.

Clinical data were available at the time of collection for 17 samples from the 10 pSLE patients. Samples from patients with a history of nephritis had lower complement component C3 levels ( 75 vs. $98 \mathrm{mg} / \mathrm{dl}$ ), but had roughly equivalent kidney function. The median serum creatinine at the time of sampling for patients with nephritis was 0.6 $\mathrm{mg} / \mathrm{dl}$ (range 0.5-0.6) and the median eGFR was $115 \mathrm{ml} /$ $\mathrm{min} / 1.73 \mathrm{~m}^{2}$ (range $108-128$ ), compared to $0.6 \mathrm{mg} / \mathrm{dl}$ (range $0.5-0.6$ ) and $110 \mathrm{ml} / \mathrm{min} / 1.73 \mathrm{~m}^{2}$ (range 108-121) in the non-renal pSLE subset. Three of the 4 were sampled while they had microscopic hematuria (5-10 rbc/hpf), but they had only minimal proteinuria (median $30 \mathrm{mg}$ / $\mathrm{dl}$ ), and none appeared to be experiencing a renal flare at the time samples were obtained.

Relationships were explored between high-titer antimatrigel Abs and routine clinical markers of kidney involvement (online suppl. table 2). High-titer IgG and IgA anti-matrigel Abs tended to be more common in patients who had hematuria. IgM was the only isotype of antimatrigel $\mathrm{Ab}$ with any degree of correlation with reactivity to double-stranded DNA and anti-matrigel IgA were significantly correlated with anti-RNP antibodies. 


\section{Discussion}

Using a single-center historical cohort of patients with a diagnosis of pSLE, we demonstrated that the measurement of anti-matrigel Ab titers by ELISA is feasible in patients as well as in mouse models of nephritis with good reproducibility. In our cohort, IgG, IgA, and IgM antimatrigel Abs all display a high specificity to diagnose SLE, and IgA anti-matrigel Abs had a high specificity for identifying pSLE patients with a history of nephritis. Although high-titers in the plasma of pSLE patients could not discriminate between pSLE patients with a history of nephritis and non-renal pSLE, IgG3 anti-matrigel titers were more sensitive than IgG, IgM, or IgA, and may warrant assessment in prospective cohorts. The presence of antimatrigel auto-Abs did not correlate with the presence of anti-dsDNA, which suggests that adding this auto-reactivity to serology panels may be of utility in monitoring disease activity in lupus.

Matrigel is a commercially available surrogate of BM, rich in extracellular matrix proteins such as laminin, collagen IV, entactin, and heparin sulfate. It is not specific for glomerular BM antigens; however, obtaining glomerular $\mathrm{BM}$ preparations from human kidney samples is laborious and would require fresh un-fixed kidney tissue that would be challenging to obtain. As a composite marker, matrigel appears to serve as the most readily available surrogate for glomerular BM antigens at this time.

The measurement of $\mathrm{BM}$ reactivity using matrigel ELISA has been previously published using one mouse model of immune complex kidney disease, the NZB/W F1 mouse [4]. Our study is important because it shows that anti-matrigel Abs also develop in a second mouse model of immune complex nephritis, the MRL/lpr model. Older female MRL/lpr mice with severe nephritis and renal failure have high titers of both anti-dsDNA Abs and anti-matrigel IgG Abs in the blood.

Only a subset of pSLE patients tested positively for high-titer anti-matrigel IgG (fig. 1b). Therefore, it is not surprising that linear mixed modeling did not find significant differences between the pSLE patients and controls. The AUC was 0.66, which is comparable to values reported for IgG anti-dsDNA Abs in SLE with specificity of 0.97 and sensitivity of 0.57 [10]. The mechanism by which only a subset of pSLE patients develop this auto-Ab response is unknown, but the proportion of pSLE patients in our cohort that tested positively was similar to the proportion of adult SLE patients reported by Li et al. [12]. The 39 adults in their cohort were recruited from an internal medicine rheumatology clinic at Albert Einstein College of Medi- cine, mean age 39 years (range 15-69), 46\% of whom had active nephritis and $56 \%$ had positive dsDNA Abs. However only one patient was $<20$ years and $74 \%$ of patients were $>30$ years. Although investigators reported a clustering of anti-matrigel IgG reactivity with IgG reactivity to vimentin, laminin, heparin sulfate, and myosin, and they showed an association between this cluster of IgG autoreactivities and SLEDAI scores $>8$, the actual association between anti-matrigel IgG and disease activity was not reported. Anti-matrigel IgM reactivity was also tested and correlated with polyreactivity against most of the auto-antigens tested including histones, chromatin and DNA.

Anti-matrigel Abs did not discriminate between pSLE patients with a history of nephritis and those with non-renal SLE, although the sensitivity of high-titer IgG3 anti-matrigel Abs for predicting a history of nephritis was $88 \%$ (table 1). These results are comparable with those of anti-dsDNA Abs, which have a $41 \%$ specificity and $65 \%$ sensitivity [10]. Based on the ability of monoclonal anti-matrigel Abs to cause disease in nonautoimmune mice [4], it is possible that these Abs may be pathogenic to the kidney, like anti-MPO in ANCAassociated vasculitis. Therefore, monitoring changes in titer of anti-matrigel Abs, as is often done for antidsDNA Abs, may provide better prediction of an upcoming renal flare.

Limitations of this study include the small sample size and the retrospective nature of the study. From the cohort of the patients for whom we were able to obtain clinical data, none of the 4 patients with a history of nephritis had active disease during the time when samples were obtained. Only 3 patients were sampled at the time of hematuria, and in most of the cases, the hematuria was mild (5-10 rbc/hpf). Similarly, only 3 of the samples were collected at the time of proteinuria. We were unable to develop precise ELISA protocols specific for detection of IgG1, IgG2, or IgG4 subsets of anti-matrigel Abs. Future studies are warranted in order to assess the utility of antimatrigel Abs or dual positivity antibodies (anti-matrigel and anti-dsDNA Abs) as biomarkers predictive of disease activity and/or response to therapy. It would be interesting to assess whether high-titer serum anti-matrigel Abs correlate with more membranous or proliferative patterns of histopathology on biopsy, predominance of capillary loop immunofluorescence staining for immunoglobulin, or predominance of sub-endothelial, intramembranous, or epi-membranous deposition of immune complexes.

In summary, anti-matrigel ELISA for IgG, IgA, and IgM can be used to measure a potentially useful and likely pathogenic new autoimmune serology in pSLE. This 
study is, to our knowledge, the first study to detect antimatrigel Abs in children with SLE, and the first to specifically assess isotypes of anti-matrigel Abs in any SLE cohort. Based on the findings of this study, anti-matrigel Abs should be considered a potential serum serologic marker that can be used in combination with other promising serum and urine biomarkers currently in development.

\section{Acknowledgments}

The authors would like to thank clinical study coordinators Theresa Falk and Brooke Bessard and the entire staff of the Dan Duncan Institute for Clinical and Translational Research (ICTR) at BCM for their assistance in this study, and Dr. Michael Braun (BCM) for his participation in thoughtful discussions and review of the manuscript.

\section{Sources of Funding}

BCM Department of Pediatrics Pilot Award Program for Translational Research, and NIH DK081663 (P.I. Wenderfer) and the Lupus Foundation of America (P.I. Eyal Muscal).

\section{Conflicts of Interest}

None of the authors had anything to declare.

\section{References}

1 Woodroffe AJ, Border WA, Theofilopoulos AN, Götze O, Glassock RJ, Dixon FJ, Wilson $\mathrm{CB}$ : Detection of circulating immune complexes in patients with glomerulonephritis. Kidney Int 1977;12:268-278.

-2 Couser WG, Steinmuller DR, Stilmant MM, Salant DJ, Lowenstein LM: Experimental glomerulonephritis in the isolated perfused rat kidney. J Clin Invest 1978;62:1275-1287.

3 Yung S, Chan TM: Anti-DNA antibodies in the pathogenesis of lupus nephritis - the emerging mechanisms. Autoimmun Rev 2008;7:317-321.

-4 Krishnan MR, Wang C, Marion TN: AntiDNA autoantibodies initiate experimental lupus nephritis by binding directly to the glomerular basement membrane in mice. Kidney Int 2012;82:184-192.

-5 Barsalou J, Levy DM, Silverman ED: An update on childhood-onset systemic lupus erythematosus. Curr Opin Rheumatol 2013;25:616-622.

-6 Sturfelt G, Truedsson L: Complement and its breakdown products in SLE. Rheumatology (Oxford) 2005;44:1227-1232.
7 Mok CC: Biomarkers for lupus nephritis: a critical appraisal. J Biomed Biotechnol 2010; 2010:638413.

8 Brunner HI, Bennett MR, Mina R, Suzuki M, Petri M, Kiani AN, Pendl J, Witte D, Ying J, Rovin BH, Devarajan P: Association of noninvasively measured renal protein biomarkers with histologic features of lupus nephritis. Arthritis Rheum 2012;64:26872697.

-9 Watson L, Beresford M: Urine biomarkers in juvenile-onset SLE nephritis. Pediatr Nephrol 2013;28:363-374

10 Kavanaugh AF, Solomon DH: Guidelines for immunologic laboratory testing in the rheumatic diseases: anti-DNA antibody tests. Arthritis Rheum 2002;47:546-555.

11 Marion TN, Lawton AR 3rd, Kearney JF, Briles DE: Anti-DNA autoantibodies in (NZB $\mathrm{X}$ NZW)F1 mice are clonally heterogeneous, but the majority share a common idiotype. J Immunol 1982;128:668-674.

12 Li QZ, Xie C, Wu T, Mackay M, Aranow C, Putterman C, Mohan C: Identification of autoantibody clusters that best predict lupus disease activity using glomerular proteome arrays. J Clin Invest 2005; 115:34283439.

13 Muscal E, Bloom DR, Hunter JV, Myones BL: Neurocognitive deficits and neuroimaging abnormalities are prevalent in children with lupus: clinical and research experiences at a US pediatric institution. Lupus 2010;19:268279.

14 Hochberg MC: Updating the American college of rheumatology revised criteria for the classification of systemic lupus erythematosus. Arthritis Rheum 1997;40:1725.

15 Schwartz GJ, Muñoz A, Schneider MF, Mak RH, Kaskel F, Warady BA, Furth SL: New equations to estimate GFR in children with CKD. J Am Soc Nephrol 2009;20:629637.

16 Wenderfer SE, Ke B, Hollmann TJ, Wetsel RA, Lan HY, Braun MC: C5a receptor deficiency attenuates $\mathrm{T}$ cell function and renal disease in MRLlpr mice. J Am Soc Nephrol 2005;16:3572-3582. 